\title{
Construction and characterization of thymidine auxotrophic ( $\triangle$ thy $A$ ) recombinant Lactobacillus casei expressing bovine lactoferricin
}

Han Zhou ${ }^{1,2+}$, Xuechun $\mathrm{Li}^{1+}$, Zongying Wang ${ }^{1}$, Jiyuan Yin ${ }^{1}$, Hongchao Tan ${ }^{1}$, Li Wang ${ }^{1,2}$, Xinyuan Qiao ${ }^{1,2}$, Yanping Jiang ${ }^{1,2}$, Wen Cui ${ }^{1,2}$, Min Liu ${ }^{3}$, Yijing Li ${ }^{1,2}$, Yigang $X_{u^{1,2^{*}}}$ and Lijie Tang ${ }^{1,2^{*}}$ (D)

\begin{abstract}
Background: Lactobacillus casei (L. casei) is well known for its probiotic property in human and animals. Lactoferricin (Lfcin) polypeptide can effectively modulate host immune responses and have antimicrobial activity in vivo and in vitro. In order to develop a food-grade L. casei system constitutively expressing bovine Lfcin, this study constructed a thymidine auxotrophy ( $\triangle$ thyA) recombinant L. casei.

Results: Based on the thymidylate synthase gene (thyA) insert site, LFEC(Lfcin expression cassette)was inserted into $L$. casei genome through homologous recombination, successfully expressed and could be stably inherited. The recombinant L. casei, $\triangle$ thyA L. casei-LFEC, is sensitive to chloramphenicol and limited when cultured without thymine. Meanwhile, $\triangle$ thyA L. casei-LFEC has both good antibacterial activity against Escherichia coli and Staphylococcus aureus and antiviral activity against porcine epidemic diarrhea virus (PEDV).

Conclusions: We successfully constructed a recombinant L. casei strain expressing Lfcin, $\triangle$ thyA L. casei-LFEC, which could only survive in the presence of thymine, and had excellent antimicrobial and antiviral activity with good genetic stability and sensitivity. This research provides a cost-effective alternative to the antibiotics with additional biological functions and wider applicability prospect. Using $\Delta$ thy $A$ as the selectable mark instead of antibiotic to construct genetic engineering L.casei provides a safe and effective approach of feed additives in livestock raising.
\end{abstract}

Keywords: Recombinant Lactobacillus casei, Thymidine auxotrophy, Expression of Lfcin

\section{Background}

Lactobacillus casei (L. casei) is well known as a kind of gram positive bacteria with its probiotic property for human and animals, which can maintain microflora homeostasis, inhibit pathogens growing and regulate $\mathrm{pH}$ balance in the host gastrointestinal environment [1]. Compared to Escherichia coli (E. coli) expression system, the most significant advantage of $L$. casei expression system is that the genetic engineered $L$. casei with vaccine and pharmaceutics purposes can be directly applied via oral administration [2-4]. However, antibiotic

\footnotetext{
*Correspondence: yigangxu_china@sohu.com; tanglijie@163.com

${ }^{+}$Han Zhou and Xuechun Li contributed equally to this work.

${ }^{1}$ College of Veterinary Medicine, Northeast Agricultural University, Chang

Jiang road No. 600, Xiang Fang District, Harbin, China

Full list of author information is available at the end of the article
}

resistances are common used as selectable mark for the construction of genetic engineering $L$. casei expression systems [5], which would result in potential risk to the environment and human.

Thymidylate synthase encoded by thymidylate synthase gene (thy $A)$ is a kind of highly conserved isozyme present in different bacteria, which plays a crucial role in DNA synthesis, and works as the key enzyme in de novo synthesis of phosphorylated deoxythymidine uracil (dTMP) by catalyzing deoxyuridine ribonucleotides (dUMP) into dTMP via methylation modification [5, 6]. When the thy $A$ is deleted, the DNA de novo synthesis pathway in $L$. casei will be blocked, resulting in proliferation failure [7]. Meanwhile, addition with thymidine or thymine in culture as

(c) The Author(s). 2018 Open Access This article is distributed under the terms of the Creative Commons Attribution 4.0 International License (http://creativecommons.org/licenses/by/4.0/), which permits unrestricted use, distribution, and reproduction in any medium, provided you give appropriate credit to the original author(s) and the source, provide a link to the Creative Commons license, and indicate if changes were made. The Creative Commons Public Domain Dedication waiver (http://creativecommons.org/publicdomain/zero/1.0/) applies to the data made available in this article, unless otherwise stated. 
the substrate for dTMP synthesis may promote the growth of L. casei [5].

Lactoferricin (Lfcin) polypeptide is dissociated off from lactoferrin under acidic condition, which can effectively modulate host immune responses, such as recruiting and promoting the balance of the production of immune cells [8]. The Lfcin has antimicrobial activity in vivo and in vitro [9]. Lfcin with positive charges could establish nonspecific binding with the lipid layer carrying negative charges in cell walls, and then induce autolysis death of bacteria cell by increasing its membrane permeability [10]. Moreover, the Lfcin could block the iron intake of microorganisms to act antimicrobial activity [11]. The Lfcin also possesses many other probiotic properties, such as antioxidation, antiviral activity, inhibiting tumor cell growth and regulating the immunity of the organism [12-16].

In this study, we successfully constructed a genetic engineering L.casei using $\triangle t h y A$ as the selectable mark instead of antibiotic, followed by the expression of bovine Lfcin as a multifunctional protein, suggesting a safe and effective approach for feed additives of livestock or in other industries.

\section{Methods}

\section{Bacterial strains, cell strain and virus strain}

Bacterial strains used in this study are listed in Table 1. L. casei and $\triangle$ thyA L. casei was cultured in GM17 broth supplemented with $40 \mu \mathrm{M}$ of thymidine at $37^{\circ} \mathrm{C}$. The temperature-sensitive plasmid pGBHCupp was constructed in our laboratory, containing a pWV01 replicon and chloramphenicol resistance genes. VERO cell line (ATCC ${ }^{\circ}$ CCL-81 $1^{\mathrm{m}}$, USA) was stored in our laboratory. PEDV LJB/03 was preserved in our laboratory at $-80^{\circ} \mathrm{C}$.

\section{Construction of the pGBHCupp-TF-LFEC-TR}

The HCE promoter, T7 g10 enhancer, signal peptide of peptidoglycanhydrolase and Myc tag, and the two segments of bovine Lfcin (LFcinB and Lfampin) connected with linker were synthesized and inserted into pUC57 vector by Genewiz Biological Technology Company, Ltd., Beijing, China. The recombinant plasmid was named pUC57-Lfcin. The terminator rrnBT1T2 gene was amplified by $\mathrm{PCR}$ using pMD18-T-HCE-MCS-rrnBT1T2 plasmid DNA as template. The rrnBT1T2 fragment was gel purified, inserted into pMD19-T-vector (Takara, DaLian, China), and named pMD19-TS-rrnBT1T2. To construct LFEC(Lfcin expression cassette), Lfcin was digested off from pUC57-Lfcin and inserted into pMD19-TS-rrnBT1T2 (Fig. 1a). The recombinant plasmid was named pMD19-TS-LFEC.

For homologous recombination in L. casei 393, upstream homologous arm TF and downstream homologous arm TR were PCR amplified using L. casei 393 genomic DNA as a template to create an internal deletion in the thyA site. The two fragments were gel purified, inserted into the pMD19-T vector and named pMD19-TS-TFTR. Then, the LFEC was digested off from pMD19-TS-LFEC, inserted into pMD19-TS-TFTR and named pMD19-TS-TF-LFEC-TR.

The TF-LFEC-TR was digested off from pMD19-TS-TF-LFEC-TR, inserted into pGBHCupp vector (Fig. 1c) and named pGBHCupp-TF-LFEC-TR, which was both temperature sensitive and chloramphenicol resistant.

PCR primers used for amplifying target genes are listed in Table 2.

\section{Electrotransformation}

Briefly, $100 \mathrm{ml}$ culture of $L$. casei cells was grown in MRS medium at $37^{\circ} \mathrm{C}$ for $4 \mathrm{~h}$. The cells were ice-bathed

Table 1 Bacterial strains and plasmids used in this study

\begin{tabular}{|c|c|}
\hline & Genotype / characteristics \\
\hline \multicolumn{2}{|l|}{ E. coli strains } \\
\hline TG1 & E. coli cloning host \\
\hline $\begin{array}{l}\text { pMD18-T-HCE-MCS-rrnBT1T2/ } \\
\text { TG1 }\end{array}$ & TG1harbring pMD18-T-HCE-MCS-rrnBT1T2,Cmr \\
\hline pGBHC-Pupp/TG1 & TG1 harboring pGBHC-Pupp, Cmr \\
\hline pUC57-LFcin/JM109 & JM109 harboring pUC57-Lfcin, Amr (Synthesis by Genewiz Biological Technology Co., Ltd) \\
\hline pGBHCupp-TF-LFEC-TR/TG1 & TG1 harboring pGBHCupp-TF-ELFEC-TR, Cmr \\
\hline \multicolumn{2}{|l|}{ L. caseistrains } \\
\hline AuppL.caseiATCC393 & L. casei ATCC393 derivative without upp \\
\hline pGBHCupp-TF-LFEC-TR/L.casei & AuppL.caseiATCC393 harboring pGBHCupp-TF-ELFEC-TR, Cmr \\
\hline$\Delta$ thyAL.casei-LFEC & $\Delta$ thyAL.casei ATCC393 with LfcinBexpression cassette insertion in the thyA \\
\hline \multicolumn{2}{|l|}{ Plasmids } \\
\hline pGBHC-upp & Ori (pWV01) with a copy of upp expression cassette, $\mathrm{Cmr}$ \\
\hline pGBHCupp-TF-LFEC-TR & $\begin{array}{l}\text { pGBHC-upp containing } L f c i n B \text { expression cassette between upstream and downstream sequence flanking the thy } A \\
\text { integration site }\end{array}$ \\
\hline
\end{tabular}




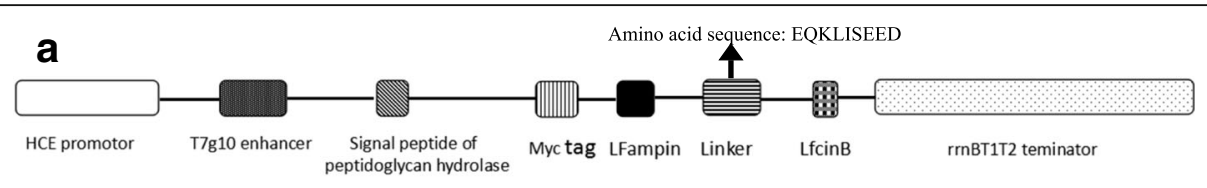

LFEC expression cassette

b

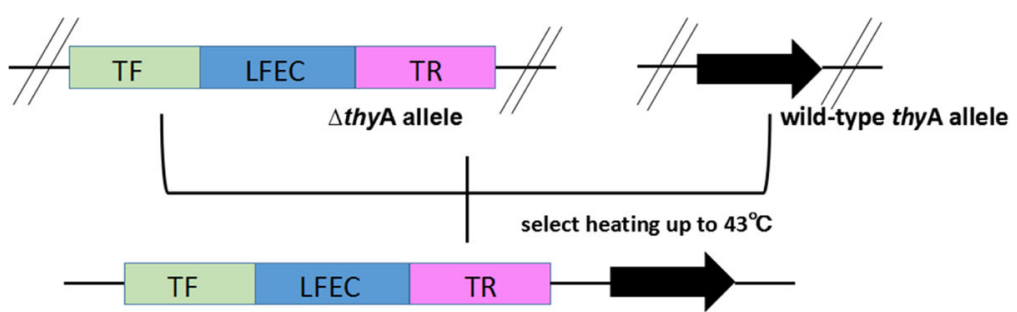

C

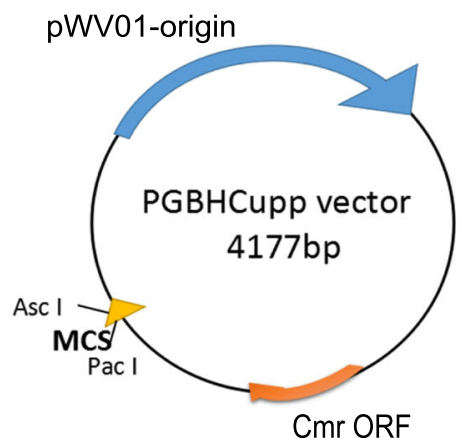

Fig. 1 Construction diagram of integration plasmids PGBHC-upp-LFEC. a Schematic diagram of LFEC. $\mathbf{b}$ Schematic diagram of the insertion of LFEC between homologous arms. The wild-type thyA was replaced by LFEC through homologous recombination, which occurs after the temperature reaches $43{ }^{\circ} \mathrm{C}$ due to the application of the temperature-sensitive plasmid. c Schematic diagram of the insertion of LFEC into pGBHCupp plasmids

Table 2 Primers used in this study

\begin{tabular}{|c|c|c|}
\hline Primer & Primer sequence $\left(5^{\prime}-3^{\prime}\right)$ & Product size \\
\hline \multicolumn{3}{|c|}{ Cloning primers } \\
\hline $\operatorname{rrnBT} 1 \mathrm{~T} 2$ & $\begin{array}{l}\text { F:ACTAGTGTCAATGATGAGATCTGGCTGTTTGGCGGATGAGAGA } \\
\text { R:CTCGAGAGAGTTTGTAGAAACGCAAAAAGGC }\end{array}$ & 438 bp \\
\hline LFEC & $\begin{array}{l}\text { F:TCTAGAGATCTCTCCTTCACAGATTCCCAAT } \\
\text { R:CTCGAGAGAGTTTGTAGAAACGCAAAAAGGC }\end{array}$ & 1022 bp \\
\hline TF & $\begin{array}{l}\text { F: GGCGCGCCTTAGGCGAGACCGTTCAT } \\
\text { R:TTAATTAACGTATGACGCACTAGTCGATCAGGCTTGAAATGG }\end{array}$ & 1020 bp \\
\hline TR & $\begin{array}{l}\text { F:ACTAGTGACTAGCGATCTCGAGCACGCATACAGGCACGTA } \\
\mathrm{R}: \text { TTAATTAAGGGTCACGAGCAAGGTAT }\end{array}$ & 1392 bp \\
\hline \multicolumn{3}{|c|}{ Screening primers } \\
\hline thyA & $\begin{array}{l}\text { F: TGTGGCATCACTTAGGACC } \\
\text { R: TGTGGCATCACTTAGGACC }\end{array}$ & $3268 \mathrm{bp}$ \\
\hline \multicolumn{3}{|c|}{ Real-time PCR primes } \\
\hline PEDV-N & $\begin{array}{l}\text { F:ACTGAGGGTGTITCTGGGTTGC } \\
\text { R:GGTTCAACAATCTCAACTACACTGG }\end{array}$ & $137 \mathrm{bp}$ \\
\hline Beta-actin & $\begin{array}{l}\text { F:AAGGATTCATATGTGGGCGATG } \\
\text { R:TCTCCATGTCGTCCCAGTTGGT }\end{array}$ & $103 \mathrm{bp}$ \\
\hline
\end{tabular}


for $10 \mathrm{~min}$, harvested by centrifugation at $5000 \mathrm{~g}$ for $10 \mathrm{~min}$ at $4^{\circ} \mathrm{C}$, and resuspended in $20 \mathrm{ml}$ of ice-cold EPWB buffer. Then, the cells were harvested and resuspended in ice-cold EPWB buffer for two more times as described above. Finally, the competent cells were harvested and resuspended in $1 \mathrm{ml}$ EPB buffer. 200 ng of pGBHCupp-TF-LFEC-TR plasmid was added to $200 \mu \mathrm{l}$ of competent cells in ice bath and transformed by electroporation at $2.0 \mathrm{kV}$. Cells were recovered in MRS medium for $2-3 \mathrm{~h}$ and then spread on MRS agar plate containing $2.5 \mu \mathrm{g} / \mathrm{ml}$ of chloramphenicol. The positive colony was identified and the recombinant pGBHCupp-TF-LFEC-TR/L.casei was obtained.

\section{Homologous recombination of $L$. casei ATCC 393}

The process of homologous recombination was followed with temperature sensitive selection (Fig. 1b). The integration transformants were transferred (1\% inoculum) three times at $43^{\circ} \mathrm{C}$ and grown to stationary phase each time to select single-crossover integrations, after which the pGBHCupp vector containing chloramphenicol resistant gene stopped replication. Then, the single-crossover integrations ( $1 \%$ inoculums) were propagated in GM17 broth for 30 generations in the absence of antibiotic resistance to achieve the second homologous crossover and lose the recombinant plasmid. The presence of the LFEC insertion in $\triangle$ thyA L. casei chromosome was detected by PCR with the primers thy $A$ F and thyA $\mathrm{R}$ shown in Table 2. The positive integration $L$. casei was named as $\triangle$ thyA L. casei-LFEC.

\section{Test of auxotrophy and chloramphenicol-sensitivity}

In order to test the dependence of $\triangle t h y A L$. casei-LFEC on thymine, the $\triangle$ thyA L.casei-LFEC was cultured overnight in GM17 broth supplemented with thymineand spread on GM17 agar plates in the presence or absence of thymine for incubation at $37^{\circ} \mathrm{C}$ for $48 \mathrm{~h}$. Then, the bacteria concentration was determined. In parallel, L.casei was used as control. In order to detect plasmids residual in $\triangle$ thyA L.casei-LFEC for chloramphenicol resistance, the bacteria cultured overnight in MRS broth supplemented with thymine was spread on MRS agar plates in the presence or absence of chloramphenicol at $37^{\circ} \mathrm{C}$ for $48 \mathrm{~h}$ to observe.

\section{Growth kinetics of the $\triangle$ thyA L.casei-LFEC}

In order to analyze growth kinetics of the $\triangle$ thyA L.case$i$-LFEC, the growth curve of the $\triangle$ thyA L.casei-LFEC was determined in the presence or absence of thymine. The bacteria density was measured by absorbance at $\mathrm{OD}_{600}$ every $2 \mathrm{~h}$ until $48 \mathrm{~h}$. In parallel, L. casei cultured in GM17 broth in the presence or absence of thymine was used as control.

\section{Stability of the $\triangle$ thyA L.casei-LFEC}

To determine the recombinant strain stability post chromosomal integration, the culture of $\triangle$ thyA L.case$i$-LFEC (1\% inoculum) was continuously transferred 50 generations at interval of $12 \mathrm{~h}$, then genomic DNA of each generation was extracted and detected by PCR for the presence of LFEC. PCR primers used in this study are listed in Table 2.

\section{Determination of Lfcin expressed by $\Delta$ thyA L.casei-LFEC}

Western blot assay was performed to analyze the expression of Lfcin in $\triangle$ thyA L.casei-LFEC cultured in GM17 broth supplemented with thymine. Following the extraction of total proteins in $12 \%$ sodium dodecyl sulfate-polyacrylamide gel electrophoresis (SDS-PAGE), the proteins were electro-transferred onto a nitrocellulose membrane, incubated with mouse anti-LfcinB antibody prepared in our lab and horseradish peroxidase (HRP)-conjugated goat anti-mouse IgG antibody diluted at 1:5000 (Invitrogen, USA), and visualized with a chemiluminescent substrate reagent according to the manufacturer's instruction $[17,18]$.

Indirect immunofluorescence assay was performed to detect the expression of Lfcin on the cell surface of $\triangle$ thyA L.casei-LFEC as previously described [19]. Briefly, $1 \mathrm{~mL}$ of $\triangle$ thyA L.casei-LFEC cultured in GM17 broth for $12 \mathrm{~h}$ was centrifuged, washed with PBS three times, and resuspended in $1 \mathrm{~mL}$ of sterile PBS-3\% bovine serum albumin (BSA) containing mouse anti-myc antibody; following the incubation at $37^{\circ} \mathrm{C}$ for $1 \mathrm{~h}$, the cells were harvested, washed three times, and incubated in $1 \mathrm{~mL}$ of FITC-conjugated goat anti-mouse IgG antibody (diluted at $1: 500$ ) at $37^{\circ} \mathrm{C}$ for $1 \mathrm{~h}$; then, the cells were washed three times, transferred onto a glass slide, and fixed with cold acetone for $30 \mathrm{~min}$; Confocal microscope was used to observe fluorescence signals. In parallel, $L$. casei was used as negative control.

The Lfcin concentration in the supernatant of $\Delta$ thy $A$ L.casei-LFEC was determined using the native bovine Lfcin (XingHao Pharmaceutical co., ltd., WuHan, China) as standard sample and anti- Lfcin monoclonal antibody (preserved in our lab) as detection antibody.

\section{Antimicrobial activity test of Lfcin}

To determin the antimicrobial activity of Lfcin, single bacterial colony of E. coli and S. aureus were inoculated in LB broth respectively, then $10 \mathrm{~mL}$ of filtered $(0.22 \mu \mathrm{m})$ supernatant of $\Delta$ thyA L.casei-LFEC was added when the $\mathrm{OD}_{600}$ value reached 0.3-0.4. In comparison, the supernatant of L.casei culture was used as negative control. Bacteria concentration of each group was detected by determining $\mathrm{OD}_{600}$ value at intervals of $2 \mathrm{~h}$. At every time point, the colony counting of E. coli and $S$. 
aureus were performed and the inhibition percentages were calculated.

\section{Transmission electron microscopy (TEM)}

To study the insight of the direct effects of Lfcin in the morphology of bacterial cells, logarithmic growth phase of $S$. aureus and E. coli cells after treatment with the supernatant of $\triangle$ thyA L.casei-LFEC were chosen to assess the bacterial membrane damage by TEM. After incubation, the cells were pelleted by centrifugation at $1000 \mathrm{rpm}$ for $5 \mathrm{~min}$, followed by washing thrice with PBS. Subsequently, the cells were fixed with $2.5 \%$ glutaraldehyde for $1 \mathrm{~h}$, washed in PBS for three times, centrifuged in an series of increasing ethanol (30, 50, 70, 90\% and absolute ethanol) 20-25 $\mathrm{min}$ [20]. The cells were penetrated with acetone and embedding agent at a 1:1 volume, shaken for $2 \mathrm{~h}$ by an oscillator (Qilinbeier, Jiangsu, China), and again shaken for $2 \mathrm{~h}$ in the pure embedding agent before polymerization in the incubator at $37^{\circ} \mathrm{C}$ for $24 \mathrm{~h}, 45^{\circ} \mathrm{C}$ for $48 \mathrm{~h}$, and $60^{\circ} \mathrm{C}$ for $48 \mathrm{~h}$. Next, $120 \mathrm{~nm}$ ultra-thin slices were sectioned and stained with $4 \%$ uranyl acetate for 20 min and with double electron staining with lead citrate for $5 \mathrm{~min}$. These ultra-thin sections were then placed on a single-hole copper mesh and were subjected to observation and photography under electron microscopy.

\section{Antiviral activity test of Lfcin}

VERO cells were grown in 96-wells tissue culture plates at $37^{\circ} \mathrm{C}$ in $5 \% \mathrm{CO}_{2}$ until $85 \%$ confluence and infected with PEDV at $1.0 \mathrm{MOI}$ for $1 \mathrm{~h}$ at $37^{\circ} \mathrm{C}$ [21]. For the experiment groups, $100 \mu \mathrm{L}$ filtered supernatant of $\Delta t h y A$ L.casei-LFEC was added to VERO cells before PEDV absorption, simultaneously with PEDV and after PEDV absorption. For the control groups, $5.0 \mathrm{ng} / \mathrm{L}$ of native bovine Lfcin was added as positive control, while the filtered supernatant of L.casei was added as negative control. VERO cells were frozen and thawed for three times at $72 \mathrm{hpi}$, after which total RNA was extracted using Fast2000 RNA kit and cDNA was gained using Reverse Transcription enzyme (Toyobo, Japan). Real-time fluorescent quantitative PCR was performed to detect the viral replication using FastStart Universal SYBR Green Master (Roche, Switzerland) and ABI 7500 real time PCR system was used to determine the viral replication.

\section{Statistical analysis}

The results were analyzed as the $\left(1 / 2^{-\Delta \Delta C t}\right) \pm \mathrm{SD}$ [21] the $\Delta \Delta C t=\left(\mathrm{Ct}_{\text {PEDV }} \mathrm{N}\right.$ gene- $\left.C \mathrm{Ct}_{\beta \text {-actin }}\right){ }_{\text {Lfcin }} /\left(\mathrm{Ct}_{\text {PEDV }} \mathrm{N}\right.$ gene- $\left(t_{\beta \text {-actin }}\right)_{\text {negative control. Comparisons between groups }}$ were performed using analysis of Tukey's. The $P$ value of $<0.05(P<0.05)$ was considered as statistically significant and $P<0.01$ as highly significant.

\section{Results}

\section{Construction of the $\triangle$ thyA L.casei-LFEC}

LFEC was inserted between the homologous arms (TF and TR) as indicated in Fig. 1b. As confirmed by PCR results (Fig. 2), following the construction of pGBHC-upp-TF-LFEC-TR/L.casei, the LFEC constitutively expressing Lfcin was successfully inserted into L.casei genome via homologous recombination.

\section{Test of auxotrophy and chloramphenicol-sensitivity of $\triangle$ thyA L.casei-LFEC}

The results showed that only pGBHCupp-TF-LFEC-TR/ L.casei (PT) could grow on the GM17 plate supplemented with $\mathrm{Cmr}$ and thymine (Fig. 3a), indicating no residual antibiotic resistance present in $\triangle$ thyA L.casei-LFEC; on the GM17 plate supplemented with thymine and without Cmr, L. casei, pGBHCupp-TF-LFEC-TR /L.casei and $\triangle$ thyA L.casei-LFEC could normally grow (Fig. 3b); on the GM17 plate without thymine and Cmr, L. case $i$ and pGBHCupp-TF-LFEC-TR/L.casei could normally grow, but not $\triangle$ thyA L.casei-LFEC (Fig. 3c). Our results indicated that the $\triangle$ thyA L.casei-LFEC without $\mathrm{Cmr}$ resistance was constructed successfully.

\section{Determination of growth kinetics of $\triangle$ thyA L.casei-LFEC} Compared to wild type L. casei, $\triangle$ thyA L.casei-LFEC could grow normally with the addition of thymine in GM17 broth. However, the growth of $\triangle$ thyA L.case$i$-LFEC was limited in the absence of thymine,

\section{LFEC expression cassettes integrated successfully into the genome of $L$.casei}

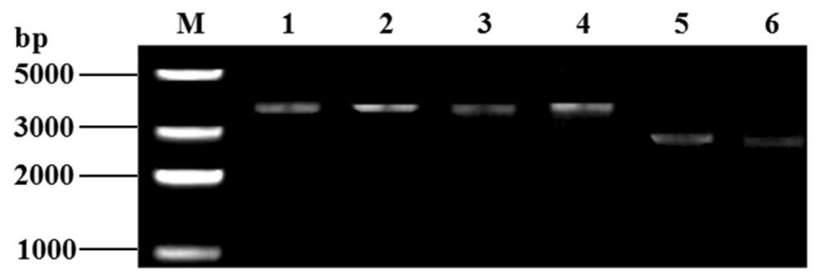

Fig. 2 Identificaiton of the insertion of LFEC into $\triangle$ thyA L. casei genome. M: DNA marker; Lanes 1-4: PCR results for $\triangle$ thyA L.casei with LFEC insertion; Lanes 5-6: PCR results for $\triangle$ thyA L. casei without LFEC insertion 
The results of auxotrophy and Cm-sensitivity of $\triangle$ thy A L.casei-LFEC
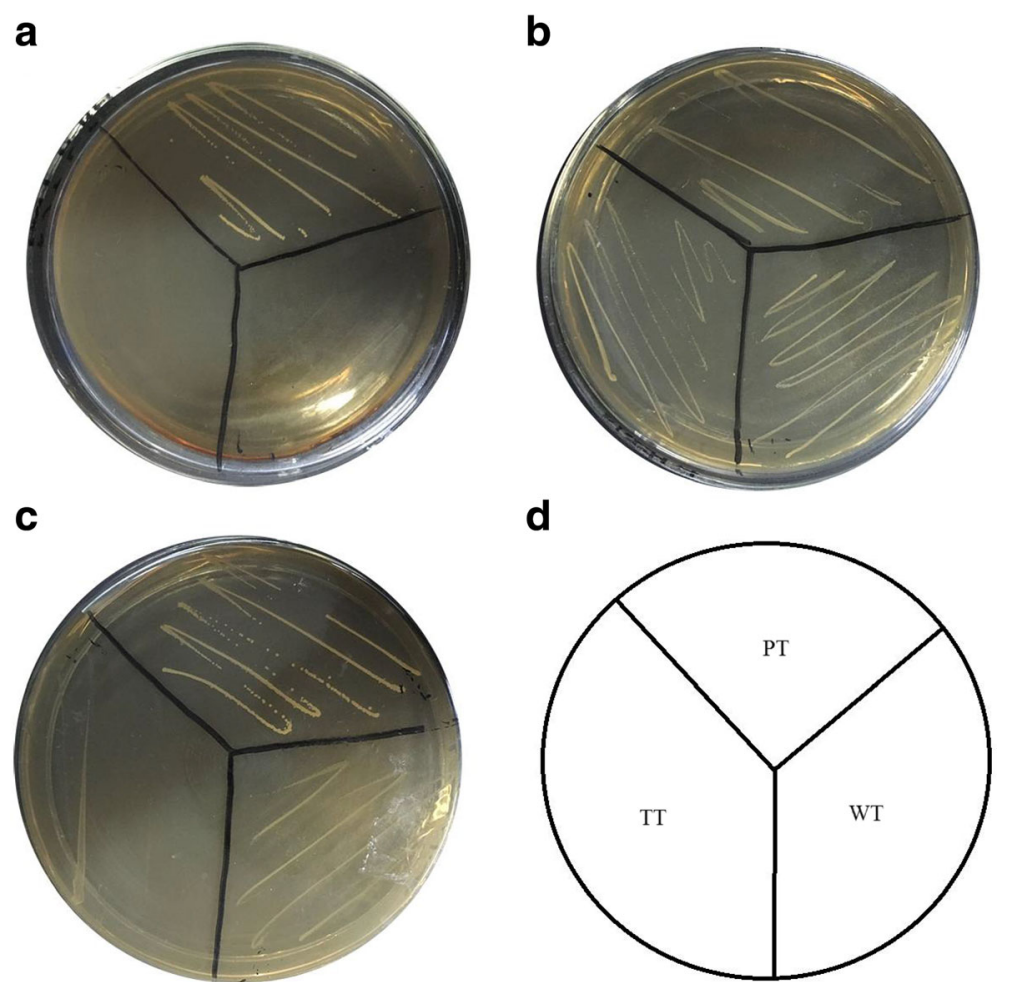

Fig. 3 Detection of thymidine auxotrophy and chloramphenicol-sensitivity of $\Delta$ thyA L.casei-LFEC. a GM17 plate containing both chloramphenicol and thymine; (b) GM17 plate containing thymine but no chloramphenicol; (c) GM17 plate containing neither chloramphenicol nor thymine; (d) Diagram of different strains: WT, L.casei; PT, pGBHCupp-LFEC/L.casei; TT, $\Delta$ thyA L.casei-LFEC

indicating the thymine dependence of $\Delta$ thyA L.casei-LFEC growth (Fig. 4).

\section{Genetic stability of $\triangle$ thyA L.casei-LFEC}

The $\triangle$ thyA L.casei-LFEC was serially cultured for 50 generations and the presence of the LFEC integrant was detected by PCR. As shown in Fig. 5, the LFEC was still detectable in the thyA location of $\Delta$ thyA L.casei-LFEC, indicating good genetic stability.

\section{Expression of Lfcin by $\triangle$ thyA L.casei-LFEC}

Western blot analysis of Lfcin expression showed an immunoblot band of expected size (Fig. 6a), while indirect immunofluorescence results showed obvious

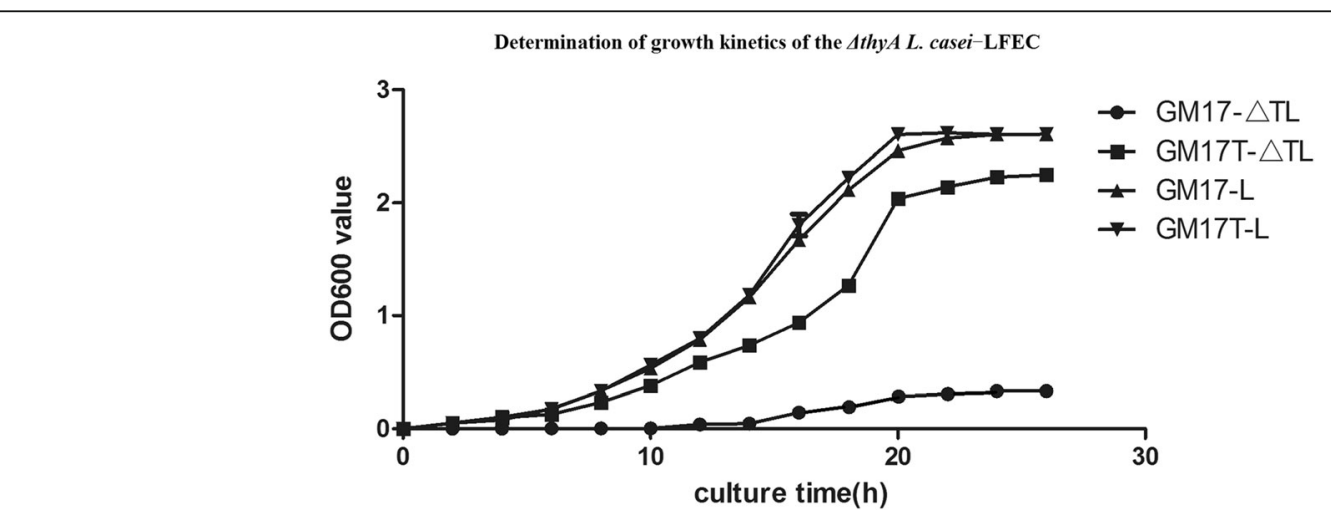

Fig. 4 Growth kinetics of $\Delta$ thyA L.casei-LFEC in different GM17 medium using L. casei as control. GM17- $\Delta T L$, growth curve of $\Delta$ thyA L. casei-LFEC in GM17 without thymine; GM17T- $\Delta T L$, growth curve of $\Delta$ thyA L. casei-LFEC in GM17 with thymine; GM17-L, growth curve of L. casei in GM17 without thymine; GM17T-L, growth curve of L. casei in GM17 with thymine 


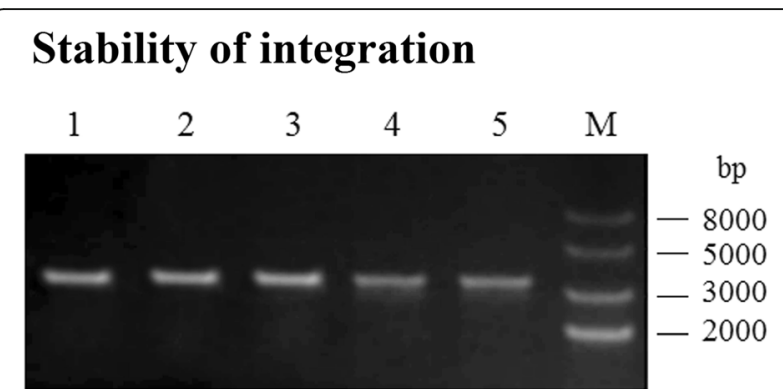

Fig. 5 Detection of the genetic stability of $\triangle$ thyA L. casei-LFEC. M: DNA marker; Lanes 1-5: PCR amplification results of LFEC of the 10 th to the 50 th generation of $\triangle$ thyA L. casei-LFEC

fluorescence on the cell surface of $\Delta$ thyA L.casei-LFEC (Fig. 6b). Both experiments confirmed the successful expression of Lfcin in $\Delta$ thyAL.casei-LFEC. The Lfcin concentration in the supernatant of $\triangle$ thyA L.casei-LFEC was determined to be $23.37 \mu \mathrm{g} / \mathrm{mL}$.

\section{Antibacterial activity of Lfcin expressed by $\Delta$ thyA L.casei- LFEC}

Compared to wild type L. casei, the growth of both $S$. aureus and E. coli were obviously limited and the inhibition percentage were 42.22 and $40.05 \%$ higher respectively when cultured with Lfcin, indicating a strong antibacterial activity of Lfcin (Fig. 7). Meanwhile, TEM results showed that Lfcin lead to damages in cell morphology of $S$. aureus and E. coli, including rough cell surface and cell lysis (Fig. 8b, d, f and h), which indicated the potential bactericidal effect of Lfcin.

\section{Antiviral activity of Lfcin expressed by $\Delta$ thyA L.casei-LFEC} The effect of Lfcin on the cellular receptors and viral proteins was tested with simultaneous incubation conditions. A significant inhibition of viral replication was observed when native bovine Lfcin or Lfcin expressed by $\Delta$ thyA L.casei-LFEC were added with viral together, compared to the negative control (Fig. 9).

\section{Discussion}

Using genetic engineered lactic acid bacteria (LAB) to deliver functional protein is a promising approach especially for oral administration development, which could effectively protect protein from protease digestion and induce effective functions [22-25]. LAB expression system has been widely used in oral vaccine development, whose effect has been proven both in vitro and in vivo. Anbazhagan et al. generated a recombinant LAB that constitutively overexpressed B. subtilis oxalate decarboxylase and degraded oxalate efficiently under in vitro conditions [26]. Moreover, Giselli et al. constructed a recombinant LAB that could secrete attenuated recombinant staphylococcal enterotoxin $B$ and induce a protective immune response in a murine model of S. aureus infection [27]. Currently, for the construction of LAB expression system, some antibiotic resistances are widely used as selection marker. However, with the application of genetic engineered LAB harboring, the

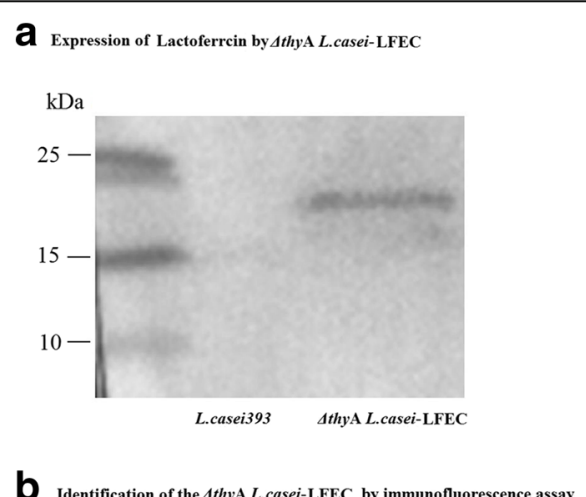

D Identification of the AthyA L.casei-LFEC by immunofluorescence assay
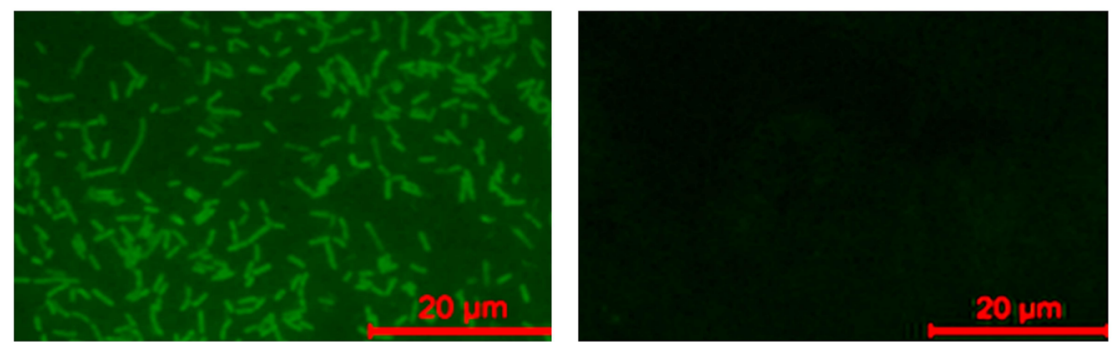

Fig. 6 Expression identification of LFcin of $\triangle$ thyA L. casei-LFEC. a Western blot identification: Left lane, marker; Middle lane, L. casei; Right lane, $\triangle$ thyA L. casei-LFEC; (b) Indirect immunofluorescence identification: Left, $\triangle$ thyA L. casei-LFEC; Right, L. casei 


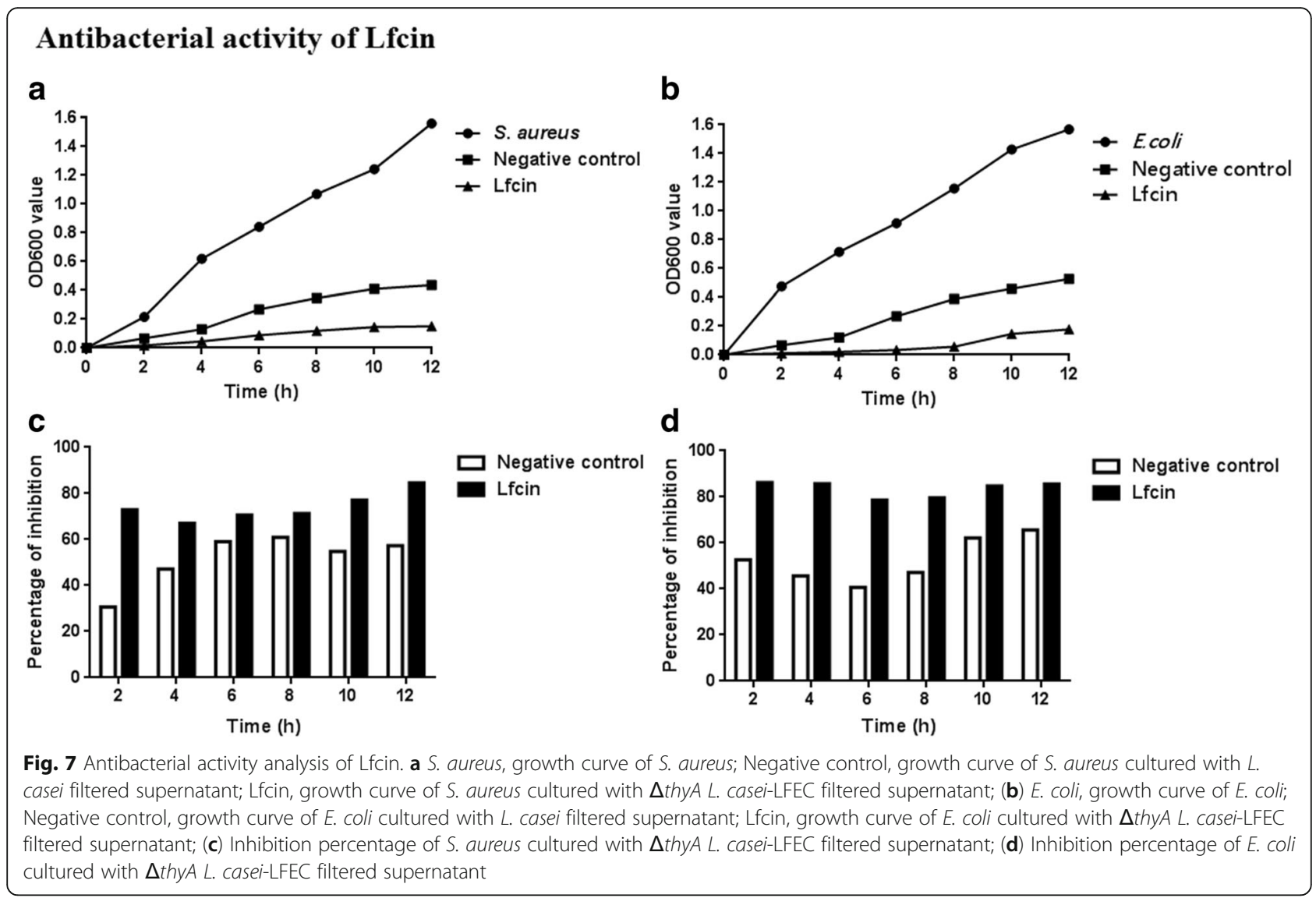

Transmission electron microscopy

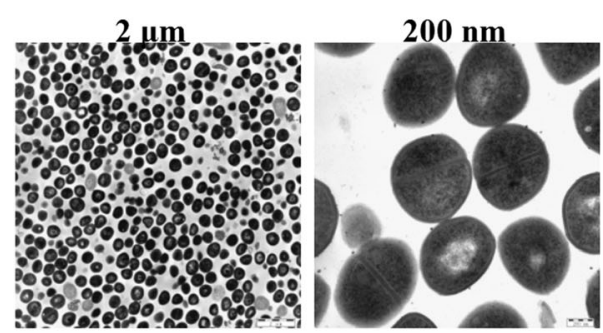

S.aureus CVCC 26003

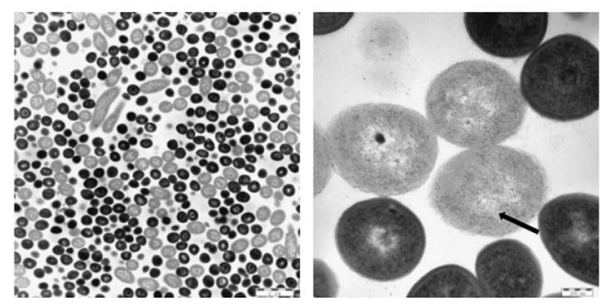

S.aureus CVCC 26003 + Lfcin

b 


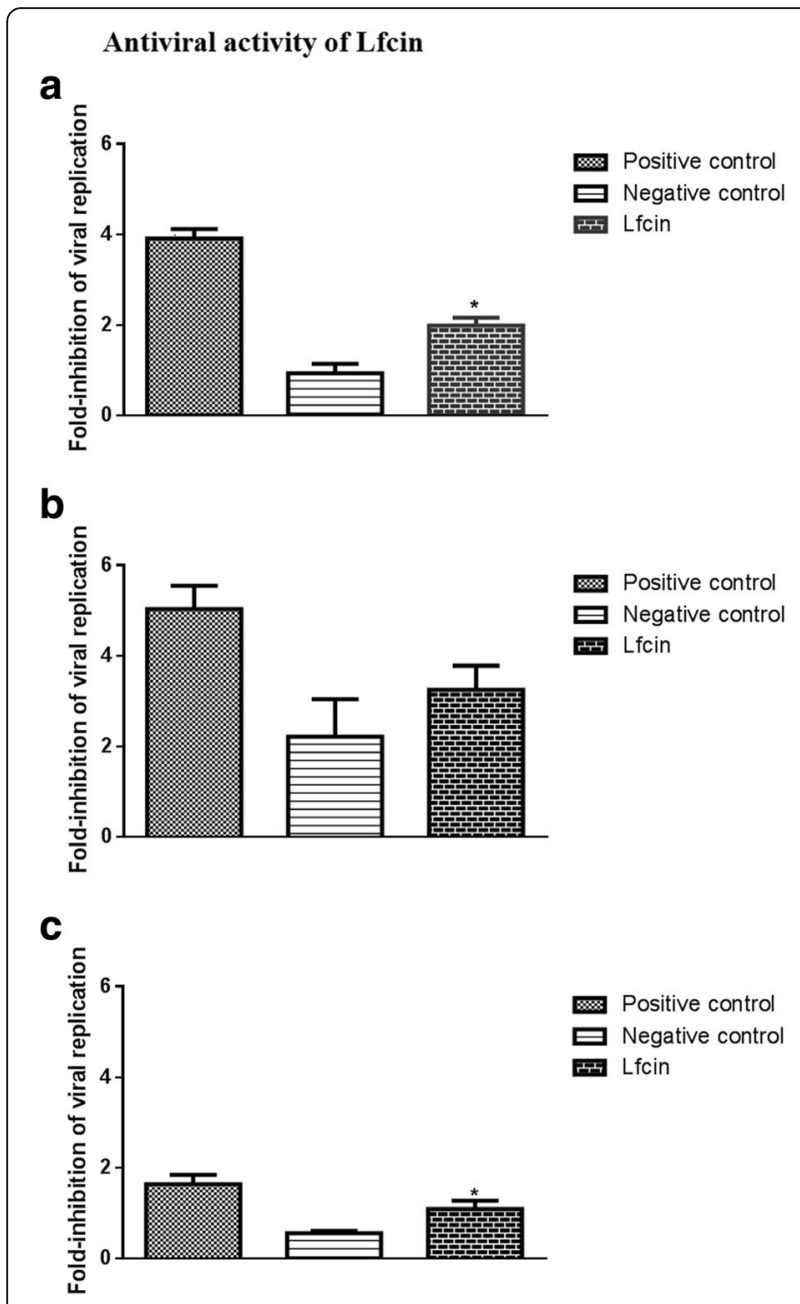

Fig. 9 Antiviral activity analysis of Lfcin expressed by $\triangle$ thyA L. caseiLFEC. Positive, the inhibition of PEDV replication with the addition of native bovine lactoferricin; Negative control, the inhibition of PEDV replication with the addition of the supernatant of cultured L. casei; Lfcin, the inhibition of PEDV replication with the addition of the supernatant of cultured $\triangle$ thyA L. casei-LFEC; (a) The supernatant was incubated with VERO cells before PEDV adsorption; (b) The

supernatant were simultaneously added to VERO cells with PEDV; (c) The supernatant were added to VERO cells after PEDV adsorption

antibiotic resistance genes would be exposed to the environment and animals, causing potential risk to human.

In this study, we successfully constructed a thy $A$-based auxotrophy genetic engineered L. casei ( $\triangle$ thyA L. case$i$-LFEC) with the deletion of antibiotic resistance gene as selection marker. The growth of thy $A$ auxotrophy $L$. casei is high limited by the additional adding thymine or thymidine in the culture media, showing high thymine dependence. Compared to the antibiotic resistance selection marker, the most significant advantage of auxotroph selection marker is the safety to the environments. Thus, the $\triangle$ thyA L. casei-LFEC constructed in this study would provide a promising approach for food-grade agents' development.

Lfcin is a kind of polypeptide with diverse biological functions, such as antibacterial effect, antiviral activity, antitumor effect and immunity modulation, suggesting a potential agent for food additive or immunologic adjuvant [28]. Bovine Lfcin was usually produced in vitro by yeast or $E$. coli $[29,30]$, while the complex purification process is required. Using lactic acid bacteriato deliver it suggested an alternative approach for utilizing Lfcin via oral administration. In the present study, by using the $\triangle$ thyA L. casei as delivery carrier and the Lfcin as the target functional protein, a genetic engineered $L$. case $i$ constitutively expressing Lfcin, $\triangle$ thyA L. casei-LFEC, was constructed through two-step homologous recombination. The recombinant $\triangle$ thyA L. casei-LFEC was passed for 50 generations and showed good genetic stability. In our previous study, wild-type L. casei has already been applied to construct chromosomal insertion strains using homologous recombination which showed similar genetic stability to this study [25].

Furthermore, the antimicrobial activity and the antiviral activity of the Lfcin were evaluated in vitro. The growth curve results of $E$. coli and S. aureus indicated that the growth of both pathogenic bacteria were significantly inhibited when cultured with Lfcin. Also, E. coli and $S$. aureus showed cell damages in morphology in TEM experiment, which confirmed the antibacterial activity of Lfcin expressed in $\triangle$ thyA L. casei-LFEC. Meanwhile, TEM results showed that Lfcin caused changes in cell membrane permeability which lead to nucleic acid area leak and the loss of bacteria pathogenesis. In addition, the antiviral effect of Lfcin on PEDV replication was evaluated by determining the inhibition fold-chage of PEDV replication in three different infection phases treated with Lfcin. Lfcin showed significantly stronger inhibition effect than negative control in the group of Lfcin treatment pre-PEDV absorption, while there was no significant difference between Lfcin and negative control in the group of simultaneous addition of Lfcin with PEDV, indicating a possibility that Lfcin might affect the susceptibility of the cell receptors to PEDV, and the affinity of PEDV with cell receptors had impact on the inhibition effect of Lfcin. It has also been reported in other studies that the block of the binding between PEDV and the cellular receptors could effectively reduce PEDV infection [31-33]. Interestingly, in the group of Lfcin treatment after-PEDV absorption, whose overall inhibition effect of three different infection phases was obviously lower than that of the other two groups, Lfcin still showed a significantly stronger inhibition effect than negative control We speculated that Lfcin might activate a different cell defense mechanism after failing to block PEDV at the phase of viral 
entry, which needs further confirmation. As negative control, the supernatant of Lactobacillus casei also showed inhibitory effect on PEDV replication, indicating unspecific antiviral activity of Lactobacillus casei which has been also proven in other reports [34-39].

\section{Conclusion}

In summary, we constructed a genetic engineered auxotrophy L. casei expressing Lfcin ( $\triangle$ thyA L.casei), which has antibacterial and antiviral activities. This research provides a safe and effective approach for oral functional protein and other pharmaceutics purposes.

\section{Abbreviations \\ Cmr: Chloramphenicol; dTMP: Deoxythymidine uracil; dUMP: Deoxyuridine ribonucleotides; E. coli: Escherichia coli; L. case: Lactobacillus casei; LAB: Lactic acid bacteria; Lfcin: Lactoferricin; PCR: Polymerase chain reaction; PEDV: Porcine epidemic diarrhea virus; SD: Standard deviation; SDS- PAGE: Sodium dodecyl sulfate-polyacrylamide gel electrophoresis; TEM: Transmission electron microscopy; VERO: Verda Reno; $\Delta$ thyA: Thymidine auxotrophy \\ Funding \\ This work was supported by the National Natural Science Foundation of China (Grant 31672461) and Technology Innovation Foundation of Harbin (Grant 2014RFXXJ084). The funders did not play any role in the design, conclusions or interpretation of the study.}

\section{Availability of data and materials}

The data analyzed during the current study are available from the corresponding author on reasonable request.

\section{Authors' contributions}

Dr. LT had full access to all of the data in the study and takes responsibility for the integrity of the data and the accuracy of the data analysis. Study concept and design: $L T, Y L$ and $Y X$. Experiment performance: $X L, Z W, H T$ and LW. Analysis and interpretation of the data: XL, ZW, HT and LW. Drafting of the manuscript: $\mathrm{HZ}$ and $\mathrm{XL}$. Critical revision of the manuscript for important intellectual content: All authors. Statistical analysis: XL, XQ, YJ. Study supervision: $L T, Y L$ and $Y X$. All authors read and approved the final manuscript.

\section{Ethics approval \\ Not applicable.}

\section{Competing interests}

The authors declare that they have no competing interests.

\section{Publisher's Note}

Springer Nature remains neutral with regard to jurisdictional claims in published maps and institutional affiliations.

\footnotetext{
Author details

${ }^{1}$ College of Veterinary Medicine, Northeast Agricultural University, Chang Jiang road No. 600, Xiang Fang District, Harbin, China. ${ }^{2}$ Heilongjiang Key Laboratory for Animal Disease Control and Pharmaceutical Development, Harbin, China. ${ }^{3}$ College of Animal Science Technology, Northeast Agricultural University, Harbin, China.
}

Received: 7 February 2018 Accepted: 5 June 2018

Published online: 27 June 2018

\section{References}

1. Gibson GR, Probert HM, Loo JV, Rastall RA, Roberfroid MB. Dietary modulation of the human colonic microbiota: updating the concept of prebiotics. Nutr Res Rev. 2004;17(2):259-75.
2. Caplice E, Fitzgerald GF. Food fermentations: role of microorganisms in food production and preservation. Int J Food Microbiol. 1999;50(1-2):131-49.

3. Gosalbes MJ, Esteban CD, Galan JL, Perez-Martinez G. Integrative food-grade expression system based on the lactose regulon of lactobacillus casei. Appl Environ Microbiol. 2000;66(11):4822-8.

4. Richters CD, Mayen I, Havenith CE, Beelen RH, Kamperdijk EW. Rat monocyte-derived dendritic cells function and migrate in the same way as isolated tissue dendritic cells. J Leukoc Biol. 2002;71(4):582-7.

5. Steidler L, Neirynck S, Huyghebaert N, Snoeck V, Vermeire A, Goddeeris B, Cox E, Remon JP, Remaut E. Biological containment of genetically modified Lactococcus lactis for intestinal delivery of human interleukin 10. Nat Biotechnol. 2003;21(7):785-9.

6. Kim EB, Son JS, Zhang QK, Lee NK, Kim SH, Choi JH, Kang SK, Choi YJ. Generation and characterization of thymidine/D-alanine auxotrophic recombinant Lactococcus lactis subsp. lactis IL1403 expressing BmpB. Curr Microbiol. 2010;61(1):29-36.

7. Giard JC, Verneuil N, Auffray Y, Hartke A. Characterization of genes homologous to the general stress-inducible gene gls24 in enterococcus faecalis and Lactococcus lactis. FEMS Microbiol Lett. 2002;206(2):235-9.

8. Siqueiros-Cendon T, Arevalo-Gallegos S, Iglesias-Figueroa BF, GarciaMontoya IA, Salazar-Martinez J, Rascon-Cruz Q. Immunomodulatory effects of lactoferrin. Acta Pharmacol Sin. 2014;35(5):557-66.

9. Latorre D, Puddu P, Valenti P, Gessani S. Reciprocal interactions between lactoferrin and bacterial endotoxins and their role in the regulation of the immune response. Toxins. 2010;2(1):54-68.

10. Hancock RE, Chapple DS. Peptide antibiotics. Antimicrob Agents Chemother. 1999;43(6):1317-23.

11. Wakabayashi H, Matsumoto H, Hashimoto K, Teraguchi S, Takase M, Hayasawa H. N-Acylated and D enantiomer derivatives of a nonamer core peptide of lactoferricin B showing improved antimicrobial activity. Antimicrob Agents Chemother. 1999;43(5):1267-9.

12. Yamauchi K, Tomita M, Giehl TJ, Ellison RT 3rd. Antibacterial activity of lactoferrin and a pepsin-derived lactoferrin peptide fragment. Infect Immun. 1993;61(2):719-28

13. Wakabayashi H, Uchida K, Yamauchi K, Teraguchi S, Hayasawa H, Yamaguchi H. Lactoferrin given in food facilitates dermatophytosis cure in Guinea pig models. J Antimicrob Chemother. 2000;46(4):595-602.

14. Britigan BE, Lewis TS, Waldschmidt M, McCormick ML, Krieg AM. Lactoferrin binds CpG-containing oligonucleotides and inhibits their immunostimulatory effects on human B cells. J Immunol. 2001;167(5):2921-8.

15. Andersen $\mathrm{JH}$, Jenssen $\mathrm{H}$, Gutteberg TJ. Lactoferrin and lactoferricin inhibit herpes simplex 1 and 2 infection and exhibit synergy when combined with acyclovir. Antivir Res. 2003;58(3):209-15.

16. Beutler B, Cerami A. Cachectin, cachexia, and shock. Annu Rev Med. 1988;39. 75-83.

17. Wang Z, Jiyuan Y, Su C, Xinyuan Q, Lijie T, Yijing L. Development of an antigen capture enzyme-linked immunosorbent assay for virus detection based on porcine epidemic diarrhea virus monoclonal antibodies. Viral Immunol. 2015;28(3):184-9.

18. Tang L, Kang H, Duan K, Guo M, Lian G, Wu Y, Li Y, Gao S, Jiang Y, Yin J, et al. Effects of three types of inactivation agents on the antibody response and immune protection of inactivated IHNV vaccine in rainbow trout. Viral Immunol. 2016:29(7):430-5.

19. Shan Z, Yin J, Wang Z, Chen P, Li Y, Tang L. Identification of the functional domain of the porcine epidemic diarrhoea virus receptor. J Gen Virol. 2015; 96(9):2656-60.

20. Debebe T, Kruger M, Huse K, Kacza J, Muhlberg K, Konig B, Birkenmeier G. Ethyl pyruvate: an anti-microbial agent that selectively targets Pathobionts and biofilms. PLoS One. 2016;11(9):e0162919.

21. Furlund $C B$, Kristoffersen $A B$, Devold TG, Vegarud GE, Jonassen CM. Bovine lactoferrin digested with human gastrointestinal enzymes inhibits replication of human echovirus 5 in cell culture. Nutr Res. 2012;32(7):503-13.

22. Li YJ, Ma GP, Li GW, Qiao XY, Ge JW, Tang LJ, Liu M, Liu LW. Oral vaccination with the porcine rotavirus VP4 outer capsid protein expressed by Lactococcus lactis induces specific antibody production. J Biomed Biotechnol. 2010;2010:708460.

23. Liu D, Wang X, Ge J, Liu S, Li Y. Comparison of the immune responses induced by oral immunization of mice with lactobacillus casei-expressing porcine parvovirus VP2 and VP2 fused to Escherichia coli heat-labile enterotoxin B subunit protein. Comp Immunol Microbiol Infect Dis. 2011; 34(1):73-81. 
24. Jiang X, Yu M, Qiao X, Liu M, Tang L, Jiang Y, Cui W, Li Y. Up-regulation of MDP and tuftsin gene expression in Th1 and Th17 cells as an adjuvant for an oral lactobacillus casei vaccine against anti-transmissible gastroenteritis virus. Appl Microbiol Biotechnol. 2014;98(19):8301-12.

25. Song BF, Ju LZ, Li YJ, Tang LJ. Chromosomal insertions in the lactobacillus casei upp gene that are useful for vaccine expression. Appl Environ Microbiol. 2014;80(11):3321-6.

26. Anbazhagan K, Sasikumar P, Gomathi S, Priya HP, Selvam GS. In vitro degradation of oxalate by recombinant lactobacillus plantarum expressing heterologous oxalate decarboxylase. J Appl Microbiol. 2013;115(3):880-7.

27. Asensi GF, de Sales NF, Dutra FF, Feijo DF, Bozza MT, Ulrich RG, Miyoshi A, de Morais K, Azevedo VA, Silva JT, et al. Oral immunization with Lactococcus lactis secreting attenuated recombinant staphylococcal enterotoxin B induces a protective immune response in a murine model. Microb Cell Factories. 2013:12:32.

28. Manzoni P, Meyer M, Stolfi I, Rinaldi M, Cattani S, Pugni L, Romeo MG, Messner H, Decembrino L, Laforgia N, et al. Bovine lactoferrin supplementation for prevention of necrotizing enterocolitis in very-lowbirth-weight neonates: a randomized clinical trial. Early Hum Dev. 2014; 90(Suppl 1):S60-5.

29. Iglesias-Figueroa B, Valdiviezo-Godina N, Siqueiros-Cendon T, SinagawaGarcia S, Arevalo-Gallegos S, Rascon-Cruz Q. High-level expression of recombinant bovine Lactoferrin in Pichia pastoris with antimicrobial activity. Int J Mol Sci. 2016;17(6):902.

30. Feng XJ, Xing LW, Liu D, Song XY, Liu CL, Li J, Xu WS, Li ZQ. Design and high-level expression of a hybrid antimicrobial peptide LF15-CA8 in Escherichia coli. J Ind Microbiol Biotechnol. 2014;41(3):527-34.

31. Sirichokchatchawan W, Temeeyasen G, Nilubol D, Prapasarakul N. Protective effects of cell-free supernatant and live lactic acid Bacteria isolated from Thai pigs against a pandemic strain of porcine epidemic diarrhea virus. Probiotics Antimicrob Proteins. 2018;10(2):383-90.

32. Botic T, Klingberg TD, Weingartl $H$, Cencic A. A novel eukaryotic cell culture model to study antiviral activity of potential probiotic bacteria. Int J Food Microbiol. 2007;115(2):227-34.

33. Aboubakr HA, El-Banna AA, Youssef MM, Al-Sohaimy SA, Goyal SM. Antiviral effects of Lactococcus lactis on feline Calicivirus, a human norovirus surrogate. Food Environ Virol. 2014;6(4):282-9.

34. Jung YJ, Lee YT, Ngo VL, Cho YH, Ko EJ, Hong SM, Kim KH, Jang JH, Oh JS, Park MK, et al. Heat-killed lactobacillus casei confers broad protection against influenza a virus primary infection and develops heterosubtypic immunity against future secondary infection. Sci Rep. 2017;7(1):17360.

35. Olaya Galan NN, Ulloa Rubiano JC, Velez Reyes FA, Fernandez Duarte KP, Salas Cardenas SP, Gutierrez Fernandez MF. In vitro antiviral activity of lactobacillus casei and Bifidobacterium adolescentis against rotavirus infection monitored by NSP4 protein production. J Appl Microbiol. 2016; 120(4):1041-51.

36. Fernandez-Duarte KP, Olaya-Galan NN, Salas-Cardenas SP, Lopez-Rozo J, Gutierrez-Fernandez MF. Bifidobacterium adolescentis (DSM 20083) and lactobacillus casei (Lafti L26-DSL): probiotics able to block the in vitro adherence of rotavirus in MA104 cells. Probiotics Antimicrob Proteins. 2018;10(1):56-63.

37. Maragkoudakis PA, Chingwaru W, Gradisnik L, Tsakalidou E, Cencic A. Lactic acid bacteria efficiently protect human and animal intestinal epithelial and immune cells from enteric virus infection. Int J Food Microbiol. 2010; 141(Suppl 1):S91-7.

38. Yu M, Qi R, Chen C, Yin J, Ma S, Shi W, Wu Y, Ge J, Jiang Y, Tang L, et al. Immunogenicity of recombinant lactobacillus casei-expressing F4 (K88) fimbrial adhesin FaeG in conjunction with a heat-labile enterotoxin a (LTAK63) and heat-labile enterotoxin B (LTB) of enterotoxigenic Escherichia coli as an oral adjuvant in mice. J Appl Microbiol. 2017;122(2):506-15.

39. Ma S, Wang L, Huang $X$, Wang $X$, Chen S, Shi W, Qiao $X$, Jiang Y, Tang $L, X u Y$, et al. Oral recombinant lactobacillus vaccine targeting the intestinal microfold cells and dendritic cells for delivering the core neutralizing epitope of porcine epidemic diarrhea virus. Microb Cell Factories. 2018;17(1):20

Ready to submit your research? Choose BMC and benefit from:

- fast, convenient online submission

- thorough peer review by experienced researchers in your field

- rapid publication on acceptance

- support for research data, including large and complex data types

- gold Open Access which fosters wider collaboration and increased citations

- maximum visibility for your research: over $100 \mathrm{M}$ website views per year

At BMC, research is always in progress.

Learn more biomedcentral.com/submissions 